\title{
Per forza di levare: matière et création dans la sculpture de Michel-Ange
}

\section{Baptiste Tochon-Danguy}

\section{(2) OpenEdition}

\section{Journals}

Édition électronique

URL : http://journals.openedition.org/appareil/2952

DOI : 10.4000/appareil.2952

ISSN : 2101-0714

Éditeur

MSH Paris Nord

Référence électronique

Baptiste Tochon-Danguy, «Per forza di levare : matière et création dans la sculpture de Michel-Ange », Appareil [En ligne], Articles, mis en ligne le 10 décembre 2018, consulté le 30 juillet 2020. URL : http:// journals.openedition.org/appareil/2952 ; DOI : https://doi.org/10.4000/appareil.2952

Ce document a été généré automatiquement le 30 juillet 2020.

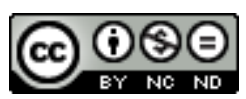

Appareil est mis à disposition selon les termes de la Licence Creative Commons Attribution - Pas d'Utilisation Commerciale - Pas de Modification 4.0 International. 


\title{
Per forza di levare : matière et création dans la sculpture de Michel-Ange
}

\author{
Baptiste Tochon-Danguy
}

1 La sculpture constitue depuis Aristote un exemple privilégié pour penser la relation de la matière et de la forme, dans la mesure où elle donne l'exemple d'une activité technique qui est une mise-en-forme d'une matière informe. Rares sont pourtant les réflexions philosophiques à avoir interrogé ce problème métaphysique en rapport avec un type de sculpture particulier, et à s'être efforcé de prendre en compte la différence qui peut exister entre la sculpture sur bronze et la sculpture sur pierre par exemple. Cette dernière, qui procède par retraits successifs de matière, peut pourtant remettre en cause cette conception du travail artistique comme mise-en-forme; et la considération du marbre, matière dure, peut produire une idée différente de la matière en général.

2 La sculpture de Michel-Ange en est une preuve privilégiée. Le sculpteur florentin a pratiqué tout au long de sa vie une technique de sculpture singulière, per forza di levare, qui consiste à retirer progressivement la matière qui entoure la forme à dégager technique qui implique que la matière ne soit pas tout à fait informe, mais possède en quelque sorte déjà la forme qu'il s'agit de mettre au jour. Chez Michel-Ange, la matière occupe donc une place ambiguë : elle est le moyen de l'œuvre finale, puisque le bloc de marbre contient la forme parfaite ; elle est également un obstacle au travail artistique, puisqu'elle dissimule cette forme. Nécessaire et indésirable, à polir et à enlever, la matière possède chez le sculpteur un double statut qui permet de poser avec acuité le problème métaphysique de la matière, à la fois disposée à la forme et réfractaire à son extraction.

Michel-Ange n'a certes pas écrit de traité théorique, bien qu'il en eût eu l'intention selon son biographe Ascanio Condivi ${ }^{1}$; mais les quelques lignes à teneur théorique que l'on trouve dans ses Rime ou dans sa correspondance font référence à ce geste de l'arte del levare, comme à une expérience décisive. Dans ces textes, Michel-Ange envisage le 
monde "sub specie sculpturae", selon l'heureuse formule de Glaucio Cambon ${ }^{2}$; l'expérience de la sculpture nous introduit à un monde matériel dur, qu'il s'agit de purifier par une opération de soustraction. Le levare est un geste exemplaire, par lequel s'exprime bien davantage qu'un procédé technique : une vision du monde, une pensée de l'être et plus précisément de la matière. Pour formuler cette pensée, qui ne se trouve pas développée dans des raisonnements déductifs et des concepts, mais enveloppée dans un geste que l'histoire de l'art reconstitue et que la poésie de Michel-Ange traduit en termes métaphoriques, nous prendrons appui sur les concepts développés par les contemporains de Michel-Ange - notamment ceux de Marsile Ficin et de Pic de la Mirandole, qui pensent la matière dans sa relation avec la forme -, qui nous garderont d'une forme d'anachronisme. Cependant, il ne s'agit pas d'appliquer les contenus philosophiques à l'œuvre sculptée, ni même de relever une quelconque influence directe. Michel-Ange a connu Ficin, Pic, Laurent de Médicis et Ange Politien à Florence lors de ses années de formation ${ }^{3}$, mais il est peu probable qu'il ait lu leurs œuvres théoriques, écrites pour la plupart en latin. En revanche, il avait une bonne connaissance du Commentaire à la Divine Comédie de Cristoforo Landino, qui abonde en références antiques et médiévales, en explications philosophiques des vers poétiques. Ses Rime et sa correspondance attestent également d'une connaissance des débats concernant la sculpture à la Renaissance et des principales œuvres poétiques de son temps. L'ensemble de ces textes théoriques ne donnent en aucun cas accès à ce qu'Eugenio Garin appelle la "pensée » de Michel-Ange ${ }^{4}$, qui est irréductible à ces théories philosophiques. Mais ils permettent d'accéder aux concepts qui «indiquent comment les expériences [des artistes] étaient formulables ", selon le mot d'André Chastel $^{5}$ : ils fournissent un contexte culturel commun, où artistes et intellectuels partagent si ce n'est des thèses, du moins des problèmes et des manières de voir. Ces sources seront d'une aide précieuse pour formuler une pensée qui, en toute rigueur, ne s'exprime que dans les œuvres visuelles.

4 Nous proposons dans cet article l'exemple d'une certaine philosophie de l'art, qui ne néglige ni le faire des artistes, ni la matérialité des œuvres, comme c'est parfois le cas ${ }^{6}$, et qui donne voix aux expériences des artistes et aux interprétations des historiens de l'art. La philosophie, en l'occurrence une philosophie de la matière, doit être mise à l'épreuve d'un geste technique singulier et historiquement défini pour être transformée et enrichie par cette confrontation. De l'analyse du geste sculptural, il convient donc de tirer des " conclusions philosophiques" ${ }^{7}$. La réflexion philosophique sera donc a posteriori, et plutôt que de présupposer une conception de la matière, nous interrogerons les œuvres elles-mêmes en tant qu'elles témoignent d'une pratique sculpturale singulière. L'interprétation philosophique suivra, mais en ayant toujours soin, lorsqu'elle confronte les œuvres sculptées et les pensées philosophiques, de marquer les limites, les points où elles ne coïncident pas; il faudra également toujours, comme l'écrit Eugenio Garin, "individualiser, autant qu'il est possible, dans les contenus des créations [de Michel-Ange], le reflet des doctrines variées qui circulaient autour de lui ${ }^{8}$ ».

$5 \quad$ Nous décrirons donc dans un premier temps la sculpture per forza di levare, pour en noter les principales caractéristiques; puis nous tirerons les implications qu'une telle pratique peut avoir pour une théorie de la matière et une théorie de la création. 


\section{La technique du relief}

6 "J'entends par sculpture (scultura) celle qu'on réalise par l'effort de retrancher (per forza di levare) : car celle qu'on réalise au moyen de l'adjonction (per via di porre) est semblable à la peinture ${ }^{9}$. " Dans une lettre à Varchi de 1547, Michel-Ange exclut de manière ferme la sculpture sur bronze de la définition de la sculpture. Il s'accorde en cela avec la plupart des théoriciens de l'art de la Renaissance, comme Alberti ${ }^{10}$. Le sculpteur modèle le bronze ${ }^{11}$, le met en forme, au sens propre de l'expression : il introduit une matière labile dans un moule, une forme préexistante et extérieure; il ajoute la forme à une matière qui en est dépourvue. À l'inverse, le sculpteur qui taille le marbre n'introduit rien mais soustrait de la matière, pour dégager une forme qui se trouve à l'intérieur du bloc de marbre. La sculpture sur bronze est, du fait de la spécificité de la matière sur laquelle elle travaille, semblable à la peinture, qui ajoute des couleurs et des lignes à la toile blanche. Dans le seul poème consacré à la sculpture sur bronze qu'a écrit Michel-Ange, le poète écrit que le «moule » est « vide de l'œuvre achevée »" au contraire le bloc de marbre est plein d'une forme déjà présente. Michel-Ange est l'un des rares sculpteurs de la Renaissance à réaffirmer aussi fermement la différence entre la sculpture comme taille sur pierre et la sculpture comme modelage ${ }^{13}$, et à se consacrer presque exclusivement à la taille sur pierre - alors que Donatello, ou plus tard Cellini travaillent à la fois le bronze et le marbre. Dans le cadre du paragone, de la rivalité entre les arts, et notamment entre la peinture et la sculpture, il accorde une importance décisive à la matérialité et considère que la dualité entre la soustraction et l'ajout tracent la véritable ligne de partage entre peinture et sculpture.

7 Michel-Ange se singularisait par une pratique très rigoureuse, que Rudolf Wittkower a appelée « la technique du relief ${ }^{14}$ ». Rappelons que les premières sculptures de MichelAnge étaient des reliefs, comme celui, inachevé, de la Bataille des centaures: les sculptures en ronde-bosse reproduisent cette image du relief, par la continuité des plans successifs, bien qu'il n'y ait pas de fond. Michel-Ange part principalement d'une face, puis travaille dans la profondeur du bloc de marbre, et progresse selon des plans graduels en dégageant la matière superflue. Vasari compare cette technique au fait de faire lentement émerger une figure en marbre couchée à l'horizontale et immergée dans un bassin d'eau ${ }^{15}$. Michel-Ange travaille parfois néanmoins sur deux faces en même temps, comme dans le cas de l'Esclave inachevé, intitulé par la critique L'Atlas. L'esclave se dégage d'un bloc de marbre dont deux des parois externes n'ont pas encore été travaillées ; seuls le plan frontal et un plan latéral ont été sculptés. La jambe droite émerge de la face latérale, selon ce même principe du relief; la jambe gauche de la face frontale. Cependant, cela ne change pas fondamentalement la nature de son faire, qui se refusait à tout ajout. Quelles sont les principales caractéristiques de cette «technique du relief»?

8 D'abord, la sculpture per forza di levare est un effort progressif et continu, un corps-àcorps constant avec la matière. Vasari, dans un texte d'inspiration michelangelesque, ridiculise les sculpteurs qui, « impatients de voir la figure en ronde-bosse tirée du bloc d'un seul coup", commettent des erreurs et se livrent ensuite à un vulgaire « rafistolage (rattopamento) $»^{16}$. Tailler dans la pierre sans rien ajouter rend le travail irréversible. Toute erreur est définitive : une mauvaise taille du marbre peut aboutir à l'abandon de l'œuvre. D'où la lenteur de la progression, et l'usage d'un outil qui n'ôte que très peu de matière à chaque fois : après avoir grossièrement dégagé à la pointe et 
au ciseau droit les premiers éclats de marbre, Michel-Ange utilise la gradine, un instrument qui permet de faire ressortir les contours en enlevant de la matière dans le sens de la latéralité. Cette manière de faire implique d'avoir conçu de manière très précise la forme à dégager : les formes de la statue étaient fixées sur des esquisses, comme on peut le voir dans les dessins préparatoires qui ont servi à l'extraction des statues de la Chapelle des Médicis, ou sur des modèles réduits, bozzetti, qui, selon Rudolf Wittkower, «lui permettaient de préciser ou d'affermir ses idées et lui servaient de référence au fur et à mesure que le travail du marbre progressait ${ }^{17} »$. Ce travail préparatoire permet à Michel-Ange d'affiner son disegno, son projet et son idée ${ }^{18}$, avant même de passer au travail sur pierre. Le travail sur modèle ne contredit pas le fait que la forme se trouve dans le marbre, puisqu'il a comme unique but de préciser la forme que le sculpteur devine dans le bloc; il a un rôle tout à fait analogue au dessin, comme Vasari le souligne: «Certains sculpteurs, peu accoutumés à tracer lignes et contours, ne peuvent dessiner sur le papier : ils préfèrent donc travailler en relief la terre ou la cire: façonnant des personnages, des animaux et autres sujets, ils réalisent ainsi l'équivalent d'un excellent dessin sur papier ou sur tout autre support ${ }^{19}$. Mais à la différence de ce qui se passera chez les maniéristes, Le Bernin et plus tard Rodin, le modèle n'est pas pour Michel-Ange le lieu de la découverte de l'idée; celle-ci est trouvée dans l'étude du bloc de marbre. D'où « l'extrémisme » de Michel-Ange, qui fait partie de ces «sculpteurs qui ont cherché leurs idées dans le matériau lui-même, le laissant prendre le pas sur leur volonté de donner forme et se mettant presque à son service $^{20} »$. C'est pourquoi Michel-Ange ne fait pas usage de la technique du report, qui consiste à produire un modèle en cire à taille réelle, puis à reporter ses proportions sur le marbre. Le report des proportions suppose de fait une traduction des effets esthétiques vus sur la cire vers le marbre, en imposant à la matière une logique qui lui est étrangère; la taille directe correspond mieux à une pensée qui souligne les possibilités inhérentes à la matière. Le modèle permet simplement de faire disparaître l'hésitation, qui n'a plus sa place au moment d'enlever la matière. La main qui enlève le marbre doit être guidée par une idée précise et ferme : le corps et l'intellect travaillent de concert : «On ne peut travailler, écrit Michel-Ange dans une lettre de 1525, avec les mains occupées à une chose et le cerveau à une autre, surtout s'agissant du marbre ${ }^{21}$. " La concentration requise par la sculpture per forza di levare fait de ce geste une activité intellectuelle à part entière; et la sculpture n'envie rien à la peinture de ce point de vue.

9 La statue, inachevée, du Saint Matthieu montre à merveille comment Michel-Ange opère. Ce marbre nous présente le saint à-demi dégagé du bloc de marbre; seuls les éléments frontaux de la figure, le torse, la face du visage présentée au spectateur, la jambe la plus saillante, et le bras, ont été dégagés de la pierre. L'on aperçoit encore, derrière ces premiers plans, le bloc de marbre dont la surface a été attaquée à la pointe. La quantité de marbre qui entoure la statue et qui n'a pas été dégagée reste très importante : en dépit de l'avancement du travail, Michel-Ange a voulu conserver une grande partie du bloc de marbre originel autour de la forme qu'il était en train d'extraire, sans doute pour pouvoir rectifier une erreur éventuelle: la tridimensionnalité est la dernière conquête de ce travail, non la première. Contrairement à ce qu'écrivait Léonard de Vinci, qui préconisait de travailler deux faces, celle de devant et celle de derrière ${ }^{22}$, Michel-Ange n'avance qu'à partir du plan frontal du bloc de marbre, sur lequel il dessine au charbon la figure, qu'il projette ensuite en profondeur ${ }^{23}$. Les formes les plus saillantes du Saint, déjà extraites par le 
travail du sculpteur, montrent une certaine perfection : le genou et la cuisse gauches du Saint Matthieu, alors même que le saint est encore à moitié embourbé dans la matière, sont presque achevés ${ }^{24}$. Tout se passe comme si la forme découverte était déjà parfaite, bien qu'elle soit encore à moitié ensevelie. Le sculpteur ne se retrouve jamais, à la manière d'un sculpteur grec archaïque, face à un volume en trois dimensions, informe et indistinct, qu'il faudrait ensuite mettre en forme, en travaillant sur sa surface; chaque membre dégagé est un membre déjà dessiné, visible et reconnaissable. Le travail de Michel-Ange est plus proche de celui d'un archéologue : il ne fait pas violence au marbre, mais se met à l'écoute de la matière, pour en dégager la forme qu'elle recèle. Il s'agit d'inventer, au sens de découvrir, une forme déjà présente et déterminée : de l'exhumer et de la faire parvenir à la lumière en enlevant la gangue qui la dissimule. Souvenons-nous que Michel-Ange avait été frappé par la découverte des statues antiques, telles que le Torse du Belvédère - statues extraites de la terre, mutilées, aux formes abîmées par le temps. Michel-Ange reproduit quelques-unes de ces caractéristiques sur certaines de ses statues ; dans son esprit, le travail du sculpteur est celui d'un homme parti à la découverte de formes cachées et ensevelies dans la pierre.

Ne pas déformer le marbre, mais protéger la forme qu'il contient : tel est le mot d'ordre de Michel-Ange. Il cherche à conserver l'unicité du bloc, en utilisant toutes les possibilités du bloc qu'il travaille : Condivi insiste sur cette prouesse michelangelesque qui consiste à toujours refuser d'ajouter quelque chose au bloc de marbre ${ }^{25}$; il aurait même laissé une trace d'inachevé au sommet de la tête du David afin de montrer qu'il avait utilisé l'ensemble du bloc, qui contenait toute la statue. Michel-Ange dut sculpter le David dans un bloc de marbre particulièrement étroit, déjà attaqué et mal dégrossi par un autre sculpteur. Or la statue achevée montre bien comment Michel-Ange, en réduisant notamment la largeur du bassin, a réussi à extraire de ce bloc difficile une forme qui lui corresponde. Sculpter une statue, voire un groupe sculptural, dans un seul bloc n'a rien d'évident; et le Bernin n'hésitera pas, pour la confection de son Longin, à avoir recourt à quatre blocs de marbre. Une telle technique, limitée à un seul bloc, a d'importantes conséquences, dont celles de contenir les mouvements dans les limites idéales du bloc de marbre et de mettre en valeur la compacité de l'œuvre, son unicité indivise : on peut « sentir, sur les œuvres achevées, les grandes formes simples du bloc dans lequel elles ont été taillées » et l'œuvre s'ordonne de l'« extérieur vers l'intérieur $\aleph^{26}$. Michel-Ange fait donc figure d'exception dans l'histoire de la sculpture, laquelle est hantée par la tentation d'outrepasser les possibilités de son médium. À l'inverse, le sculpteur florentin valorise les qualités propres du matériau.

11 Enfin, la technique du relief, appliquée à la ronde-bosse, implique de travailler le bloc dans la profondeur. Michel-Ange n'atténue pas le caractère imposant et massif des blocs de marbre, mais en fait des qualités esthétiques : le poids du marbre accentue l'impression de déséquilibre du Bacchus, et, dans la Chapelle des Médicis, les statues de Julien et Laurent de Médicis, qui dépassent de leur niche et sont en retrait par rapport aux autres statues, possèdent une profondeur supérieure à leur largeur, de sorte que, même vues de loin, elles s'imposent par leur matérialité. Comme l'écrit Panofsky, la "technique du relief» de Michel-Ange n'aboutit pas «à sacrifier la puissance du volume à l'harmonie du dessin à deux dimensions ${ }^{27}$, mais au contraire met en valeur ce volume dans l'espace. La forme n'est pas conquise par une lutte contre les propriétés de la matière: une fois achevée, l'œuvre rappelle toujours, par sa profondeur et l'impression de lourdeur qu'elle dégage, sa dimension matérielle. 
Le travail de Michel-Ange suit les indications que lui fournit la matière, comme celui du bon boucher chez Platon suit les articulations de la bête qu'il découpe. La matière, le marbre, n'est pas qu'un médium, l'élément dans lequel l'œuvre est créée : elle n'est pas "ce en quoi l'œuvre est faite », mais «ce dans quoi elle est extraite ». Cette originale manière de faire a d'importantes implications pour une théorie philosophique de la matière.

\section{Une matière vivante et pleine de formes}

L'artiste excellent ne possède aucune idée

(concetto)

Qu'un marbre seul ne circonscrit en soi

De sa gangue (superchio), et à cette idée parvient seulement

La main qui obéit à l'intellect (intelletto).

Michel-Ange ${ }^{28}$

Ce quatrain célèbre de Michel-Ange fait écho de manière frappante à la manière singulière dont il sculpte. L'idée ou forme - ces mots traduisent l'italien concetto, qui désigne ici à la fois ce qui est visé par un acte de l'intelligence et ce qui est présent dans le marbre - n'est pas produit isolément par l'esprit, puis appliqué artificiellement à une matière vierge. Elle n'est découverte qu'à la fin du travail du marbre, et l'intelligence doit plutôt deviner quel est le concetto que cache tel ou tel bloc de marbre, pour pouvoir créer l'œuvre la plus parfaite. Comment comprendre que Michel-Ange conçoive le concetto comme intérieur au bloc de marbre?

Benedetto Varchi, savant florentin contemporain de Michel-Ange, interprète cette thèse à la lumière de la distinction aristotélicienne entre la puissance et l'acte ${ }^{29}:$ le concetto est en puissance dans le marbre, mais en acte dans l'intelligence de l'artiste: celui-ci doit faire passer le concetto dans le marbre de la puissance à l'acte. Cette interprétation, toutefois, ne rend pas compte du fait que le concetto est conçu comme adéquat au marbre, qu'il possède une forme déterminée et qu'il est déjà présent dans le bloc de marbre. Au contraire, la forme en puissance est, dans la tradition aristotélicienne, souvent considérée comme indifférente à la matière, indéterminée (la puissance étant puissance d'être comme de non-être) ; elle est à réaliser, et non déjà réelle.

Il faut donc comprendre autrement la présence de la forme dans la matière. Davantage qu'une possibilité, la puissance signifierait une force déjà présente; or, c'est à considérer la puissance comme une tendance vers la forme qu'aboutissent les réflexions du néoplatonisme florentin. Ficin propose ainsi une « conception dynamique de la matière ${ }^{30}$ »; pour lui, la puissance de la matière est une véritable force, vis : «On appelle une telle puissance matière la plus commune, c'est-à-dire la force capable de recevoir également toute sorte de formes (vim formarum omnium aequaliter receptricem $)^{31}$. " La pensée de Ficin ne livre pas la clé de la pensée michelangelesque, dans la mesure où cette dernière prend pour objet une matière précise, et déjà en partie informée - puisque le bloc de marbre, à la différence de la matière première, possède déjà la forme du marbre et celle du bloc -, là où Ficin cherche à penser la matière commune à tous les corps, matière première avec laquelle Dieu crée l'ensemble des formes. Cependant, caractériser la matière comme une force, une capacité de 
recevoir des formes - et non certes de les produire, comme c'est le cas, à des degrés différents, pour Dieu, l'âme du monde ou l'âme humaine -, c'est déjà insister sur le fait qu'elle est prédisposée à la forme. Ficin va même parfois plus loin :

Toutes les formes sont dans la matière. Là elles sont encore appelées raisons essentielles, précisément parce qu'elles sont des stimulants, ou bien des dispositions, ou bien des tendances dirigées vers des formes (fomites sives habitudines quaedam sive respectus ad formas) qui accompagnent de manière perpétuelle la matière. Ces puissances de formes sont, dis-je, des puissances capables de formes, non efficaces, et en quelque manière des formes mêmes, mais imparfaites ${ }^{32}$.

En un sens, la matière possède des formes, mais celles-ci demeurent imparfaites : elles ne sont que des dispositions, des suggestions qui s'offrent à l'action vraiment productrice et efficace de l'âme humaine. Que la matière soit informe ne signifie pas qu'elle soit dépourvue de toute forme, mais simplement de formes accomplies et parfaites. À plus forte raison, les matières qui possèdent déjà une qualité, comme le marbre, et qui composent la nature inorganique, peuvent être considérées comme pleines de ces formes imparfaites ${ }^{33}$. Ficin opère un rapprochement conséquent entre la forme et la matière, en réinterprétant la puissance comme tendance.

17 La pensée de Ficin reste sans doute en deçà des audaces de la sculpture de MichelAnge: loin de considérer les formes présentes dans le bloc de marbre comme imparfaites, il voulait précisément les sauvegarder. C'est que Ficin ne remet jamais en cause l'idée que le travail du sculpteur est une mise-en-forme, un ajout d'une forme à une matière informe, car il pense toujours une matière labile, à l'image de la cire: "L'art produit ses œuvres selon cet ordre, à savoir qu'il imprime dans une certaine matière qui est le sujet telles et telles formes, dont la matière ne possède aucune en propre $^{34}$.» Imprimit : dans ce verbe tient toute la différence entre Ficin et Michel-Ange, pour qui frapper la pierre ne signifie pas y déposer une marque nouvelle, mais bien soustraire un superflu pour dégager une forme déjà présente.

La proximité entre le philosophe et le sculpteur est bien plus étroite lorsque Ficin confère à ce dynamisme de la matière une tonalité vitaliste. Reprenant la théorie de l' inchoatio formarum, selon laquelle sont présents dans la matière les commencements des formes, Ficin écrit :

Mais puisque se situe au-delà de l'ordre des formes cette matière première des choses qui est informe, dans laquelle se cachent (latent), pour ainsi dire, les semences des formes qui se répandent en se multipliant (formarum pullulantium semina), le rôle de l'intelligence, qui est délimitée par les formes, n'embrasse pas ces choses informes. Cependant, la matière elle-même est en une certaine mesure bonne, parce qu'elle désire le bien, c'est-à-dire la forme, parce qu'elle est disposée à accueillir le bien, parce qu'elle est nécessaire au monde bon. Les semences aussi sont bonnes, parce qu'elles sont des commencements de formes bonnes (formarum bonarum inchoationes) $)^{35}$.

Ce texte semble très proche des thèses plotiniennes sur la matière : informe, la matière ne peut être comprise par l'intelligence, laquelle ne saisit que des formes déterminées. Cependant la matière est pleine de semences qui constituent des commencements de forme, et qui sont bonnes; et plutôt que d'affirmer que la matière, puisqu'elle tend vers les formes, est dépourvue de formes et donc mauvaise, Ficin souligne ici que cette proximité de la matière à la forme rend la matière bonne. Les métaphores traduisent une forme de continuité entre les différents niveaux de réalités : tout est parcouru par un même souffle vital. C'est donc à une requalification de la matière que nous assistons : celle-ci, tout en demeurant le dernier degré de la schala naturae, tout en 
restant extérieure à l'être, participe du monde créé par Dieu et manifeste, du fait de la présence en elle de raisons séminales, une beauté qui est l'indice de sa bonté. Or cette idée d'une matière vivante et dynamique qui suggère des formes fait écho de manière étonnante aux figures inachevées de Michel-Ange, qui semblent naître à la lumière, émerger de la matière. Le travail de l'artiste est proche de celui que Ficin attribue au poète Orphée, mû par l'amour : non pas un travail d'impression, mais d'explicitation d'une forme implicite et latente :

Puisque, comme nous l'avons montré, le désir de propager sa propre perfection, inscrit en toute chose, explicite la fécondité cachée et implicite (latentem et implicatem cuiusque fecunditatem explicat), étant donné qu'il pousse les semences à se multiplier en germes, et éduit du sein de chaque chose sa force, et en conçoit la progéniture (vires cuiusque eius educit sinu fetusque concipit) et l'ouvrant comme avec des clefs il amène ce qui a été conçu jusqu'à la lumière ${ }^{36}$.

Non pas introduire une forme, mais l'éduire - la tirer du sein de la matière. Les métaphores vitales s'ajoutent aux métaphores liées à l'engendrement. Le geste du levare est bien présenté dans les Rime comme un geste érotique : c'est un geste d'amour qui consiste à développer les possibilités de la matière, à faire passer la forme des ténèbres à la lumière. Nous trouvons ici une formulation plus adéquate de la présence de la forme dans la matière. Le concetto se trouve réellement dans le bloc de marbre, non pas simplement en puissance, car il y est de manière implicite : il est à la fois dissimulé et suggéré par une matière vivante. L'implicite est précisément le nom d'une présence réelle qui se dissimule tout en étant pressentie. Au sculpteur d'expliciter l'implicite au mieux, et de rendre plus vivant ce qui l'est déjà.

Or peu de sculptures font autant voir, au sein de l'espace défini d'un bloc de marbre, le dynamisme de la matière que celle de Michel-Ange. En témoigne Vasari: «il leur a donné [aux figures], comme on l'a déjà dit tant d'art, de grâce (grazia) et tant d'une certaine vivacité (vivacità) (soit dit sans offenser personne) qu'il a vaincu et surpassé les antiques $^{37}$ ». La grâce est précisément ce qui s'ajoute à la beauté quand les attitudes et les mouvements sont vivants. La technique du relief permet, par le travail continu de la gradine, de faire ressortir les reliefs, de « définir et de redéfinir les formes naturelles, de rendre les plus subtiles modulations des corps, des muscles, de la peau et des traits du visage ${ }^{38}$ » : les plus beaux exemples en sont la fine musculature du dos du Bacchus, celle des torses du David ou des statues des Ducs de Médicis. Michel-Ange fait se rejoindre technique de sculpture et connaissances anatomiques, la dissection n'étant qu'une autre manière d'ouvrir la matière. Il utilise toutes les possibilités de la matière pour traduire le mouvement, ou du moins l'instant qui précède le mouvement comme c'est le cas du Moïse, où la partie inférieure du corps, active, contraste avec la position contemplative du haut du corps. Panofsky, dans sa thèse d'habilitation consacrée à Michel-Ange, remarquait un conflit entre une tendance à mettre en valeur la force liante du plan ainsi que la limite cubique des blocs de marbre et la volonté d'accentuer la liberté plastique de figures prêtes à se mouvoir ${ }^{39}$. Cette tension exprime le désir qu'avait Michel-Ange de montrer le dynamisme au sein de la matière, de souligner la vie de la pierre elle-même. Ce qu'il commentait dans ses Rime par une heureuse formule : «Dans la seule pierre vivante (pietra viva)/ L'art veut qu'en ce lieu vivent/ Autant que les ans le visage de ma mie ${ }^{40}$.» 


\section{La matière réfractaire et le temps de l'effort} qu'elle contient : le marbre est une matière inanimée, qui ne possède pas de puissance efficace. Du reste, la forme, pour être explicitée, demande un long et progressif effort, qui suscitait les moqueries de Léonard de Vinci :

Je ne trouve entre peinture et sculpture d'autre différence que celle-ci : le sculpteur fait ses œuvres avec plus d'effort physique (fatica di corpo) que le peintre; et le peintre les siennes avec plus d'effort intellectuel (fatica di mente). Cela se démontre, car le sculpteur doit, en produisant son ouvrage, faire un effort manuel, frappant pour enlever le superflu du marbre, ou de la pierre quelle qu'elle soit, qui dépasse la figure enfermée en son sein; ce qui exige un exercice tout mécanique, s'accompagnant souvent de beaucoup de sueur qui se mêle à la poussière et devient une croûte de boue; il a le visage tout enduit et enfariné de poudre de marbre, semblable à un boulanger, et il est couvert de petites écailles comme s'il avait neigé sur lui ; son logis est sale et plein d'éclats et de poussière de pierre ${ }^{41}$.

Texte ironique et drôle, où affleure cependant l'autre face de la matière : celle-ci résiste au travail du sculpteur. Si elle cache la forme, c'est bien à travers une gangue qui se transforme, sous les coups de marteau, en poussière et saleté. Pour être retirée, cette matière demande un effort corporel, sur lequel insistent tous les biographes de MichelAnge.

Cette résistance de la matière s'exprime dans les Rime, par la récurrence de l'expression pietra dura. Comme l'écrit Bachelard, «dur et mou sont les premiers qualificatifs que reçoit la résistance de la matière, la première existence dynamique du monde résistant ${ }^{42}$ ». Dans un poème, Michel-Ange situe cette dureté au niveau de la gangue, qui fait obstacle à la découverte et à l'explicitation du concetto implicite dans le marbre : il évoque une " écorce fruste, rude et dure ${ }^{43}$ ", dans une comparaison entre la femme aimée et le bloc de marbre. Il y a donc une partie de la matière, que nous voyons encore à l'arrière de la figure du Saint-Matthieu, que Michel-Ange avait l'intention de réduire, de soustraire et non de sauvegarder. D'abord par un travail rapide à la pointe, afin de dégager le gros du superchio, puis par un lent travail à la gradine, pour faire émerger les formes. L'inachèvement de la plupart des œuvres de Michel-Ange témoigne de la difficulté du travail qui ne pouvait pas toujours être poursuivi jusqu'à son terme. Mais cette dureté de la matière est le corrélat nécessaire de la présence de la forme en elle : le bronze ne possède ni la dureté du marbre, ni cette forme intrinsèque. Bien plus, elle peut aisément se retourner en avantage, puisqu'elle confère aux œuvres une forme de durabilité. Argument précieux pour ceux qui défendent la primauté de la sculpture sur la peinture dans le fameux paragone, comme le rappelle Varchi : « [Les partisans de la sculpture] convoquent également l'argument de la longueur du temps, en affirmant que la sculpture est presque perpétuelle ${ }^{44}$ ». Idée que Ficin formule à un niveau métaphysique par l'insistance sur la permanence de la matière : "Lorsque l'existence commune cesse, la forme par laquelle vit cette existence commune périt également. Mais la matière ne périt pas, puisqu'aussitôt elle prend une autre forme pour pouvoir exister ${ }^{45}$ ». La matière est le fond indestructible qui rend possible tout changement. Inscrire la forme dans la matière, c'est la faire participer de la nature, qui possède une force conservatrice ${ }^{46}$. Tailler la pierre, c'est aussi œuvrer pour l'éternité : la matière oppose son indestructibilité fondamentale à l'homme. Les figures de Julien et de 
Laurent de Médicis, dont Michel-Ange a fait le tombeau, semblent figées pour toujours dans cette matière dure où elles semblent vivantes.

Mais surtout, la dureté de la matière impose un certain temps au travail de l'artiste. Sur cette question, les sources hésitent : elles tendent tantôt à mettre en valeur la patience de Michel-Ange, tantôt sa facilité d'exécution : tantôt la lenteur de son travail, tantôt sa rapidité. C'est qu'un travail long et laborieux ne constitue pas, pour les hommes de la Renaissance, une qualité dont on pourrait se vanter. "Ars est celare artem ", écrivait Ovide, et Balthasar Castiglione écrit, dans son texte célèbre sur la spezzatura - la désinvolture, ou l'art de dissimuler l'effort intentionnel et les peines - que «ce qui ne paraît pas être de l'art constitue le véritable art; et on ne doit porter d'attention à rien d'autre qu'à cacher l'art, car s'il est découvert, cela enlève à toute chose sa valeur et à tout homme l'estime qu'on lui porte ${ }^{47} \nRightarrow$. Mais les sculpteurs avaient bien conscience que cet effort corporel pouvait susciter l'estime, et notamment distinguer la sculpture de la peinture; ainsi s'exprime Michel-Ange dans sa lettre à Varchi de 1547: «si un jugement, des difficultés, des entraves et un labeur considérables, ne confèrent pas une plus grande noblesse, la peinture et la sculpture sont une seule et même chose ». L'éventualité d'une supériorité de la sculpture en raison de la nature de son faire est évoquée, mais mise de côté afin d'insister, de manière consensuelle, sur l'identité des deux arts, qui font appel à une "même intelligence $»^{48}$. La sculpture de Michel-Ange manifeste, dans le marbre, une même ambiguïté. D'une part, Michel-Ange est connu pour avoir complété le geste du levare par un geste qui en effaçait les traces : celui de polir le marbre jusqu'à ce que les marques de la gradine disparaissent.

Jamais sculpteur, écrit Vasari à propos de la Pietà de 1499, ni artiste excellent ne put imaginer mettre dans une œuvre plus de grâce et de dessin, ni travailler le marbre avec cette finesse, ce poli (pulitezza) que l'on voit dans l'œuvre de Michel-Ange ${ }^{49}$.

De l'autre, il est connu pour avoir laissé des traces de non-finito sur la plupart de ces œuvres : le buste du Brutus, mais également la majorité des figures de la Chapelle des Médicis; qu'on observe les visages du Jour, où le non-finito confère une expressivité inédite au personnage.

Ce non-finito possède différents sens ${ }^{50}$. Il $\mathrm{y}$ a un non-finito contraint, qui concerne l'ensemble de l'œuvre inachevée, comme dans le cas des Esclaves destinés au tombeau de Jules II ; il renvoie au temps nécessaire au sculpteur pour accomplir son travail : les travaux de Michel-Ange ont été souvent interrompus, non seulement du fait des problèmes politiques auxquels il s'est trouvé mêlé, mais aussi en raison de la nature de son faire, qui impliquait une temporalité longue. L'inachèvement de la forme marque alors la difficulté qu'il y a à l'expliciter d'une matière dure et réfractaire. Mais il y a aussi un non-finito plutôt intentionnel, qui ne concerne pas l'ensemble de l'œuvre, mais se manifeste, de près, dans des traces d'inachèvement. Cette fonction esthétique du nonfinito était identifiée comme telle à la Renaissance ; Michel-Ange ne fut pas pour rien dans la propagation de ce goût pour l'esquisse, l'inachevé. Vasari, après la mort de Michel-Ange, ajoute un paragraphe essentiel à la Vie de Lucca della Robbia, sculpteur du Quattrocento :

[...] l'expérience enseigne que tout ce qui est vu de loin, que ce soit une peinture, une sculpture, ou quelque autre chose semblable, a plus d'effet et de force si elles sont une belle esquisse que si elles sont finies; et, en dehors de cet effet produit par la distance, il apparaît également que, dans les esquisses, s'exprime souvent l'idée (concetto) en peu de coups, naissant d'un coup d'un délire artistique (furore dell'arte), et que, au contraire, l'effort et la trop grande application enlèvent de la force et du savoir à ceux qui ne savent jamais ôter les mains de l'œuvre qu'ils réalisent ${ }^{51}$. 

que la matière implique une sorte de délai. L'intelligence peut penser à la fois plusieurs réalités, mais la main ne peut réaliser le concetto découvert par l'intelligence que de manière successive, ce que le travail à la gradine accentue encore davantage. Comme l'écrit Ficin, l'âme " parce qu'elle est changeante et possède quelque chose du temps, s'accorde avec la matière et fait descendre les formes reçues d'en haut en elle-même jusqu'à la nature changeante et temporelle ${ }^{53}$ ". L'âme est une réalité intermédiaire : n'étant pas une réalité spatiale, elle peut penser différentes idées en même temps et se rapproche des réalités supérieures; toutefois, si l'âme peut agir dans la matière, c'est dans la mesure où elle peut se déployer dans le temps. Bien que, dans ce texte, le travail de l'âme consiste à retranscrire les formes intelligibles dans le sensible, on peut penser que son activité est d'autant plus temporelle lorsque l'âme doit dégager une forme présente dans le sensible. De plus « tout corps accomplit nécessairement son opération dans un intervalle de temps ${ }^{54}$ ", de sorte que la "main» de Michel-Ange devait se mesurer elle-aussi à cette temporalité qui distingue l'âme humaine, unie à un corps, de l'âme angélique ou de Dieu. Le temps que la matière impose au sculpteur avait donc une signification métaphysique et théologique. Bien que la matière tende vers la forme, la suggère, elle résiste au geste du levare par sa dureté, et lui confère une temporalité spécifique.

\section{Quelle place pour la création ?}

Si le concetto se trouve déjà dans la matière, qui le suggère, le geste du sculpteur se réduit-il à vaincre la résistance de la matière par un effort de longue haleine ? Que reste-t-il du génie de Michel-Ange, si le concetto n'est pas créé, mais simplement découvert? La sculpture de Michel-Ange s'oppose à toute conception de la création comme création ex nihilo, production de nouveau à partir de rien. Il ne s'agit pas simplement de dire que le sculpteur doit toujours créer avec un matériau initial : ce matériau a des caractéristiques, des spécificités dont le sculpteur doit tenir compte. Bien plus, la matière exerce une contrainte essentielle sur la forme finale de l'œuvre, comme le cas du David nous le rappelle. Michel-Ange serait anti-moderne : il s'oppose par avance à toute conception romantique du génie créateur, qui imprime une forme inédite à une matière, ou tire de la matière ce qu'elle semblait ne pas pouvoir livrer. Comme l'écrit Yves Hersant: «Les formes que l'artiste matérialise ne préexistent pas seulement dans son intellect mais dans la matière elle-même : impossible de concevoir une idea que la matière ne contienne. Suivant cette perspective [...], même le plus 
autonome des artistes reste tributaire d'un donné : actualisant un potentiel, il n'ajoute rien à une Création déjà parfaite ${ }^{55}$. » Contre certaines théories de la Renaissance ellemême selon lesquelles l'artiste doit parfaire la nature, lui ajouter ce dont elle manque ${ }^{56}$, la sculpture de Michel-Ange s'affirme comme une collaboration avec la matière, qui ne fait que manifester la gloire du créateur, véritable responsable de la présence du concetto dans le bloc de marbre. Le marteau de Michel-Ange n'est qu'une pâle copie du marteau divin :

Si mon grossier marteau de durs rochers

Tire telle ou telle forme d'image humaine,

De l'agent qui le tient, guide et manie,

Tirant son élan, il suit les pas d'autrui.

Mais le marteau divin qui au ciel demeure

Les autres et soi-même orne de sa course ;

Et si nul marteau sans marteau ne peut être,

De ce vif outil tout autre procède.

Or comme le coup est d'autant plus puissant

Qu'il s'élève plus haut dessus l'enclume,

Bien plus haut, celui-ci au ciel s'est envolé.

Dès lors mon œuvre, inachevée, échouera,

Si le divin forgeron or ne l'aide

Lui, qui était unique au monde ${ }^{57}$.

Il y a comme une relation de participation entre le marteau du sculpteur et le marteau divin : le marteau de Michel-Ange est l'image du marteau divin, dont il procède et dont il tire toute l'efficace. Chez Michel-Ange, toute création est nécessairement divine; et, en dépit des présentations élogieuses de Vasari, qui l'appelle le "divin Michel-Ange ", ce dernier semble vivre la comparaison avec Dieu comme la manifestation d'un écart, d'une distance irréductible, tragique.

L'artiste, pourtant, actualise un potentiel; il développe les semences présentes dans la matière, explicite l'implicite. Si la création n'est pas le passage du non-être à l'être, elle est celle d'un être enveloppé, latent, à un être lumineux et plus vivant. Tout est question de degré. Le concetto est suggéré par la matière, la force de la main conférée par la force divine, le furore dell'arte inspiré par Dieu. Mais un écart demeure entre le donné inscrit dans la matière et la forme explicitée; et dans cet écart se joue la production d'une nouveauté. Un problème analogue se pose chez Ficin : celui-ci pense non seulement qu'il y a des formes inscrites dans la matière sous la forme de semences, mais aussi que nos idées sont des dispositions innées qui fournissent les modèles des idées présentes en acte dans l'intelligence. Et pourtant, parce qu'autant les semences présentes dans la matière que celles inscrites dans notre esprit ne sont que des dispositions virtuelles, des suggestions, la contrainte laisse une marge de liberté - et offre la possibilité de l'émergence de l'inédit dans un développement qui semblait tout tracé. Évoquant l'âme de la sphère, Ficin écrit :

Aussitôt que la puissance d'un genre d'une semence prévaut sur tous les autres, l'imagination elle-même, qui est attachée à cette puissance inférieure, songe à cette semence, la désire, l'explicite dans le ciel, la forme dans les éléments, la sème dans le ciel, l'engendre dans les éléments. [...] Et ne pense pas que des effets si nouveaux dans le monde et des figures si variées dans le ciel soient explicités à chaque moment, si ce n'est par l'intermédiaire d'affections de l'âme variées et nouvelles ${ }^{58}$.

Expliciter des formes inchoatives présentes de la matière n'exclut pas toute forme d'innovation. Cette innovation est à relier à la puissance créatrice de l'âme, par laquelle, à partir de notions innées qui ne sont que des modèles, «nous fabriquons 
(fabricamus) quand il nous plait les espèces, en nous servant de l'intellect agent, comme d'un art qui nous est propre et innés9 ". Même si le concetto michelangelesque est " comparable à une Idée divine ${ }^{60}$ ", reçue par inspiration plutôt que produite, il possède également ce statut de modèle, de sorte qu'il est aussi le lieu d'une innovation, qui se donne à voir dans les dessins préparatoires : nous y voyons le sculpteur en train de chercher la forme parfaite, d'inventer, par la variation des postures, le concetto qui correspondra le mieux à son projet. Ainsi Panofsky comprenait-il le concetto comme " une représentation qui crée librement son propre objet et peut ainsi constituer un modèle permettant de créer les formes extérieures ${ }^{61} »$. Reste à souligner que cette création ne se fait qu'à partir de commencements de formes présentes et suggérées par la matière, mais aussi inscrites par Dieu dans l'esprit de l'artiste: cette liberté ne se déploie qu'au sein de contraintes bien déterminées, comme le montre le geste de Michel-Ange, lequel refusait la grande liberté offerte par le modelage ${ }^{62}$. Pour Vasari, l'innovation de Michel-Ange réside ainsi dans la production d'une double différence : différence par rapport aux anciens, puisque par ses œuvres « inimitables », il a " vaincu et surpassé les antiques ${ }^{63} »$; et différence par rapport à ses propres œuvres, dans la mesure où Michel-Ange "n'a jamais produit d'œuvre qui en rappelât une autre des siennes, parce qu'il se souvenait de tout ce qu'il avait fait ${ }^{64} »$.

Reste néanmoins une difficulté. L'innovation ne peut se faire que dans la perspective de la découverte de la forme idéale dans le marbre. Telle est véritablement l'originalité de Michel-Ange : penser la création comme une manière de mettre en valeur la matière, un donné préalable. Les dessins préparatoires témoignent tout autant d'un mouvement créatif que d'une recherche du concetto qui corresponde le mieux aux formes suggérées par le marbre. Tout le problème, à la fois théorique et pratique, est de réconcilier la conception par l'intelligence de la forme et la découverte de l'idée dans la matière, de franchir «l'abîme qui existe entre l'Idée et la réalité65 ». Le levare est la réponse technique à ce problème, puisqu'il est le geste où la main parvient à la forme que l'intelligence a discernée dans le marbre, mais c'est un travail d'une grande difficulté. Le David est l'exemple même d'une telle réussite; mais l'inachèvement des œuvres ultérieures montre que l'adéquation entre l'esprit et la matière, la main et l'idée n'est jamais assurée: tout le drame de Michel-Ange est de ne pas pouvoir égaler la production intellectuelle du concept à la richesse idéale de la matière. Mais MichelAnge ne rend jamais la matière responsable de cet écart : « Tout comme en la plume et l'encre réside/ le style bas, élevé ou moyen/ et dans le marbre image noble ou vile,/ selon qu'en sait extraire notre esprit ${ }^{66} »$. La cause essentielle de l'échec est bien l'insuffisance du concetto. Ou plutôt son caractère disproportionné, puisque l'échec est souvent dû à une forme d'ubris ${ }^{67}$, l'artiste dépassant par la force de son esprit les possibilités de la matière. Et Vasari de conclure : « les choses qu'il avait en idée étaient telles que, ne pouvant rendre avec ses mains de si grandes et de si terribles conceptions, souvent il a abandonné des œuvres commencées, et brisé tant d'autres ${ }^{68}$ ». La nouveauté que la création apporte dans son explicitation d'une forme déjà présente entraîne toujours un risque.

34 Ceci nous introduit à la signification proprement éthique du geste créateur de MichelAnge - dimension que sa correspondance souligne : dans une lettre à Luigi del Riccio de 1542, il écrit : « La peinture et la sculpture, le travail et la probité m’ont ruiné [...]. Il aurait mieux valu que dans mes premières années je me sois mis à faire des allumettes ; je ne serais pas dans de tels tourments ${ }^{69}$. $)$ Travailler la matière, c'est travailler sur soi- 
même, et l'expérience de la sculpture est une expérience de soi, une mise à l'épreuve de forces intimes. On connaît le texte de Plotin, qui fait de la sculpture le modèle de la purification et de l'élévation de soi :

Retourne en toi-même et vois. Et si tu ne vois pas encore ta propre beauté, fais

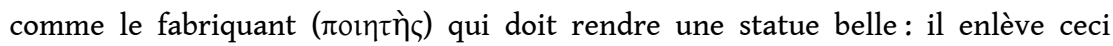

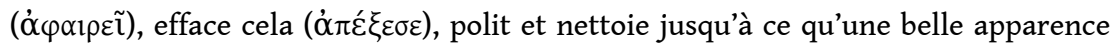

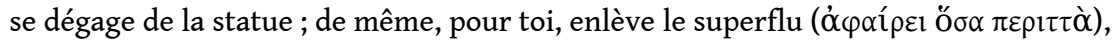
redresse ce qui est tordu et, purifiant tout ce qui est ténébreux, travaille à être resplendissant. Ne cesse de sculpter ta propre statue jusqu'à ce que brille en toi la splendeur divine de la vertu [...]..$^{70}$

Chez Plotin, la sculpture n'est purifiante que par analogie ; chez Michel-Ange, le levare, qui dégage la forme pure de sa gangue, explicite les tendances de la matière et porte à la lumière l'œuvre cachée, est immédiatement une épreuve de soi, de ses forces et de ses faiblesses. C'est bien la subjectivité qui se ressaisit dans la difficulté du travail, la matière renvoyant le sculpteur à son propre être. On peut soutenir une telle interprétation sans anachronisme, puisque l'humanisme florentin voyait dans l'expression d'une subjectivité propre l'un des enjeux de l'art, comme le montre ce passage d'une lettre d'Ange Politien: "N'exprime pas Cicéron, a dit quelqu'un. Quoi alors? en effet je ne suis pas Cicéron ; cependant, je m'exprime moi-même. [...] laisse de côté cette angoisse qui vise à te façonner seulement en tant que Cicéron et éprouve enfin tes propres forces en entier ${ }^{71}$.»Par sa résistance et sa dureté, la matière est l'occasion d'une mise à l'épreuve des forces propres. Et la sculpture est, plus que tout autre art, l'occasion d'une mise à l'épreuve conjointe du corps et de l'esprit.

Le sculpteur se métamorphose dans l'épreuve de la matière; il y recherche une élévation, qui le fasse accéder à une autre réalité, se rapprocher de Dieu. Métamorphose toujours inachevée, là est le tragique. Cependant cette métamorphose du sujet est une métamorphose de la matière, car sous les coups du ciseau et de la gradine, elle développe ses propres forces, - conformément à une théorie de l'expression élaborée à la Renaissance, dans laquelle, en vertu d'une structure en miroir, la matière est purifiée en même temps que le sculpteur. Citons Ficin :

En revanche, dans les peintures et les édifices, le dessein et l'habileté de l'artiste resplendissent. En outre, la disposition et, pour ainsi dire, une certaine figure de l'âme elle-même (quasi figura quaedam animi) se proposent au regard. De fait, l'esprit s'exprime et se reflète (exprimit et figurat) dans les ouvrages de ce genre, comme le visage d'un homme qui se regarde dans un miroir se reflète lui-même dans le miroir. [...] Dans ces ouvrages, c'est intégralement que la disposition de l'âme et la volonté se dessinent de manière absolument parfaite (tota mentis dispositio et voluntas planissime designatur), les œuvres de l'artiste ont l'habitude d'exciter en nous un affect tel que l'artiste l'a éprouvé [...]. ${ }^{72}$

Le disegno apparait dans l'œuvre achevée, et pourrait-on ajouter à partir de Vasari, encore davantage dans l'œuvre inachevée. Sculpter la matière c'est se contempler soimême ; et chercher la forme qu'elle recèle, c'est chercher sa propre forme. Michel-Ange semble bien considérer la sculpture sur pierre comme un itinéraire éthique, où le sujet peut tout autant se retrouver que se perdre. Parce qu'elle implique la durée et ses aléas, la matière est une aventure, une conquête de la forme qui n'est jamais assurée. Cependant, la signification éthique se double d'une signification cosmologique et théologique. Pic de la Mirandole, dans une lettre à Laurent de Médicis, emploie une très belle expression pour évoquer le travail du poète : « [...] toi, tu t'élèves vers le haut sur les ailes de ton génie, malgré la résistance de la matière qui te tire vers le bas, tu t'y 
portes de telle façon que tu ne te défais pas de la matière, mais l'élèves avec toi ${ }^{73}[\ldots]$. ॥ Ne pas se défaire de la matière, mais l'élever avec soi : toute l'exigence est là. MichelAnge ne veut pas simplement exhiber son talent : car les prouesses techniques ne sont valables que si elles respectent et purifient la matière elle-même, la transforment en une réalité supérieure en en révélant la qualité intrinsèque.

Ficin attribuait à l'homme une très haute fonction : «[...] l'âme de l'homme restaure un monde déjà ébranlé, puisque par son action, le monde, autrefois spirituel, mais qui a déjà été transformé en corps, est sans cesse purifié et chaque jour devient spirituel ${ }^{74}$ ». Au regard de la sculpture de Michel-Ange, sans doute faut-il nuancer cette thèse : la matière, où la forme n'est qu'esquissée, parvient-telle à accéder à un niveau de réalité plus élevé par la sculpture ? La fin de la vie de Michel-Ange, ses œuvres inachevées, ses ultimes poèmes trahissent une forte hésitation quant à la réussite d'une telle entreprise. Le groupe de la Victoire oppose, avec la figure du jeune homme qui s'appuie sur le vieillard, une matière resplendissante et déployée, à une matière enveloppée et non-finita. Ce groupe révèle un contraste entre deux moments du travail sculptural, deux niveaux de réalités, deux formes de l'humain. Significativement, c'est le vieillard qui se trouve au niveau du spectateur : tout se passe comme si s'affirmait finalement la conscience du chemin à parcourir. La sculpture per forza di levare, dont l'analyse mobilise, d'une manière typique à la Renaissance, les niveaux esthétique, métaphysique, éthique, cosmologique et théologique, témoigne des paradoxes de la matière, qui suggère la forme et résiste au travail d'extraction, et de la création, découverte d'un donné préalable et invention périlleuse.

\section{Conclusion}

L'étude philosophique du geste sculptural de Michel-Ange montre qu'il n'y a pas de dichotomie entre la matière et la forme. La matière possède une forme, le sensible est déjà spirituel ; la création n'ajoute pas à la matière, mais développe cette spiritualité initiale. La sculpture part d'une matière déjà en partie spirituelle et aboutit à une matière encore plus spirituelle. La distinction entre la matière et l'idée n'est pourtant pas abolie. Là est l'apport de la Renaissance : de mettre en valeur une continuité sans effacer les différences et les hiérarchies. C'est que la résistance de la matière au travail d'extraction de la forme permet de penser des caractéristiques essentielles du faire sculptural : sa temporalité, sa dimension corporelle, sa signification éthique. À terme, considérer la matière comme une force et le travail du sculpteur comme un processus temporel de collaboration avec la matière tend cependant à remettre en cause les divisions trop tranchées du schéma hylémorphique ${ }^{75}$. C'est à partir d'une manière de travailler une matière singulière, et non en partant d'une théorie générale de la matière qu'on appliquerait à l'art, que nous pouvons ébaucher une nouvelle philosophie de la matière. 


\section{BIBLIOGRAPHIE}

Alberti Leon Battista, La Statue, Paris, Éd. Rue d'Ulm Musée du quai Branly, 2011.

Arasse Daniel, «L’index de Michel-Ange », Communications, 1981, vol. 34, n 1, p. 6-24.

Bachelard Gaston, La Terre et les rêveries de la volonté, Paris, José Corti, 1947.

Barocchi Paola, Trattati d'arte del Cinquecento fra Manierismo e Controriforma, Bari, G. Laterza e figli, coll. « Scrittori d'Italia », 1960.

Barocchi Paola, Scritti d'arte del Cinquecento, Milan, R. Ricciardi, 1971, vol. 3.

Barolsky Paul, « As in Ovid, So in Renaissance Art », Renaissance Quarterly, 1998, vol. 51, n 2, P. ${ }^{\circ} 451-474$.

Cambon Glauco, La poesia di Michelangelo: furia della figura, trad. Paola Ternavasio, Turin, G. `Einaudi, 1991.

Castiglione Baldassare, Giovanni Della Casa et Benvenuto Cellini, Opere, Milan, R. Ricciardi, 1960.

Cellini Benvenuto, Traités de l'orfêvrerie et de la sculpture, trad. Léopold Leclanché, Paris, ENSB-A, coll. « Beaux-arts histoire », 1992.

Chastel André, Art et humanisme à Florence au temps de Laurent le Magnifique : études sur la Renaissance et l'humanisme platonicien, Paris, Presses universitaires de France, 1961 [1950].

Chastel André, Fables, formes, figures, Paris, Flammarion, coll. « Idées et recherches », 1978, vol. 2.

Combronde Caroline, «Les platoniciens de l'art à la Renaissance », Revue Philosophique de Louvain, 1999, vol. 97, n 2, p. 268-288.

Condivi Ascanio, Vie de Michelangelo Buonarroti, trad. Bernard Faguet, Castelnau-le-Lez, Climats, 1997.

Dagron Tristan, « Ficin et la conception néoplatonicienne de la matière », Marsile Ficin ou Les mystères platoniciens: actes $d u 42^{e}$ colloque international d'études humanistes [Centre d'études supérieures de la Renaissance], Tours, 7-10 juillet 1999, Stéphane Toussaint (dir.), Paris, Les Belles Lettres, 2002, p. 163-177.

Ficin Marsile, Théologie platonicienne de l'immortalité des âmes, trad. Raymond Marcel, Paris, Les Belles Lettres, 1964, 3 vol.

Ficin Marsile, Commentaire sur le Banquet de Platon, trad. Raymond Marcel, Paris, Les Belles lettres, coll. « Les Classiques de l'humanisme », 1956.

Garin Eugenio, Prosatori latini del Quattrocento, Milan, R. Ricciardi, 1952.

Gontier Thierry, « Noétique et poièsis : L'idea dans la Theologia platonica de Marsile Ficin ", Archives de Philosophie, 2004, nº 1, p. 5-22.

Hersant Yves, « Le marteau de Michel-Ange », Communications, 1997, vol. 64, n 1, p. 77-87.

Hildebrand Adolf, Le Problème de la forme dans les arts plastiques, trad. Beaufils Éliane, Paris, L'Harmattan, coll. « Esthétiques », 2002.

Ingold Tim, Making: Anthropology, Archaeology, Art and Architecture, Londres, Routledge, 2013.

Léonard de Vinci, Traité de la peinture, trad. André Chastel, Paris, Calmann-Lévy, 2003. 
Jacqueline Lichtenstein, Les raisons de l'art : essai sur les théories de la peinture, Paris, Gallimard, 2014.

Michel-Ange, Poésies = Rime, trad. Adelin Charles Fiorato, Paris, Les Belles Lettres, coll. «Bibliothèque italienne », 2004.

Michel-Ange, Carteggio = Correspondance, trad. Adelin Charles Fiorato, Paris, Les Belles lettres, coll. « Bibliothèque italienne », 2010, 2 vol.

Migliaccio Luciano, « Poemas de mármore: Michelangelo escultor e poeta nas Lezioni de Benedetto Varchi », Revista Brasileira de História, 1998, vol. 18, n 35, p. 207-216.

Panofsky Erwin, Essais d'iconologie : thèmes humanistes dans l'art de la Renaissance, trad. Claude Herbette, Paris, Gallimard, coll. « Bibliothèque des sciences humaines », 1967.

Panofsky Erwin, Idea : contribution à l'histoire du concept de l'ancienne théorie de l'art, trad. Henri Joly, Paris, Gallimard, coll. « Tel », 1989.

Panofsky Erwin, Die Gestaltungsprincipien Michelangelos, besonders in ihrem Verhältnis zu denen Raffaels, Gerda Panofsky (ed.), Berlin, De Gruyter, 2014.

Pic de la Mirandole Jean, Commento, Lausanne, L'Âge d'homme, coll. « Contemplation », 1989.

Pic de la Mirandole Jean, Euvres philosophiques, trad. Olivier Boulnois et Giuseppe Tognon, Paris, Puf, coll. «Épiméthée », 2004.

Plotin, Traités. 1-6, trad. Francesco Fronterotta, Jérôme Laurent, Laurent Lavaud et Alain Petit, Paris, Flammarion, 2002.

Rogers Leonard Robert, Comprendre la sculpture, trad. Arnaud Guilloux et Gérard Le Don, Rennes, Presses Universitaires de Rennes, 2015.

Schauder Silke, « Figures de l'inachèvement : Michel-Ange et Camille Claudel », Topique, 30 octobre2008, $n^{\circ} 104$, p. 173-190.

Snyder James G., «The Theory of materia prima in Marsilio Ficino's Platonic Theology », Vivarium, 2008, vol. 46, n 2, p. 192-221.

Summers David, Michelangelo and the language of art, Princeton, Princeton University Press, 1981.

Vasari Giorgio, Les Vies des meilleurs peintres, sculpteurs et architectes, trad. André Chastel, Paris, Berger-Levrault, 1981, vol. 1.

Vasari Giorgio, «Vie de Michel-Ange », Vies des artistes : vies des plus excellents peintres, sculpteurs et architectes, trad. Léopold Leclanché et Charles Weiss, Paris, B. Grasset, coll. « Les Cahiers rouges », 2007.

Vitale Errico, «Sul concetto di materia nella Theologia Platonica di Marsilio Ficino », Rinascimento, 1999, vol. 39.

Wittkower Rudolf, Qu'est-ce que la sculpture ? : principes et procédures, Paris, Macula, coll. « Histoire de l'art », 1995.

\section{NOTES}

1. Ascanio Condivi, Vie de Michelangelo Buonarroti, trad. Bernard Faguet, Castelnau-le-Lez, Climats, 1997, p. 159. 
2. Glauco Cambon, La poesia di Michelangelo: furia della figura, trad. Paola Ternavasio, Turin, G. Einaudi, 1991, p.87. André Chastel a proposé de voir dans ces poésies un «journal de l'affectivité de l'artiste en rapport étroit avec les hantises et les émotions de sa vie créatrice ", cf. André Chastel, Art et humanisme à Florence au temps de Laurent le Magnifique: études sur la Renaissance et l'humanisme platonicien, Paris, Puf, 1961 [1950], p. 508-509. Il ne s'agit donc ni d'un traité théorique sur la sculpture, ni d'un traité technique ou pratique, mais d'une traduction en termes poétiques de ce que pouvait signifier l'expérience sculpturale. Or cette signification nous importe car elle éclaire l'expérience que le sculpteur fait de la matière.

3. James Hankins, "Michelangelo and the culture of Laurentian Florence ", in Humanism and platonism in the Italian renaissance. II, Platonism, Rome, Ed. di storia e letteratura, 2004, p. 486.

4. Dans son article «Il pensiero di Michelangelo" (in C. Tolnay, U. Baldini, R. Salvini et al., Michelangelo: artista, pensatore, scrittore. Novare, Istituto geografico de Agostini, 1965, p. 529-541), Eugenio Garin procède à une critique des interprétations réductrices qui font de Michel-Ange luimême un penseur néoplatonicien.

5. André Chastel, Fables, formes, figures, Paris, Flammarion, coll. «Idées et recherches », 1978, vol. 2, p. 34.

6. Le constat est fait par Jacqueline Lichtenstein, dans Les Raisons de l'art : essai sur les théories de la peinture, Paris, Gallimard, 2014, p. 25.

7. Ibid., p. 171.

8. Eugenio Garin, «Il pensiero di Michelangelo », in Michelangelo: artista, pensatore, scrittore, op. cit., p. 531 (nous traduisons l'italien).

9. Michel-Ange, Carteggio = Correspondance, trad. Adelin Charles Fiorato, Paris, Les Belles lettres, coll. «Bibliothèque italienne", 2010, 2 vol., vol. 2, p. 66. Cette lettre à Varchi constitue une réponse à des Lezzioni que ce dernier prononçait sur la question du paragone.

10. Ainsi s'exprime Alberti dans le De Statua: «D'autres y sont parvenus [à exprimer dans la matière l'image qui leur plaît] par le simple fait de retirer (detrahentes) de la matière, comme ceux qui, en frappant sur le superflu (superflua), amènent à la lumière la figure d'homme qu'il recherche, qui est enfouie et cachée à l'intérieur du marbre (quaesitam hominis figuram intra marmoris glebaminditam atque absconditam producunt in lucem). Ceux-là, certes, nous les appelons sculpteurs ", Leon Battista Alberti, La Statue, Paris, Éd. Rue d'Ulm Musée du quai Branly, 2011, p. 62. Nous traduisons.

11. On trouve une description de la fonte du bronze chez un spécialiste de cette technique, Benvenuto Cellini. Cf. Traités de l'orfèvrerie et de la sculpture, trad. Léopold Leclanché, Paris, ENSBA, coll. « Beaux-arts histoire », 1992.

12. " vota d'opera prefetta », Poésies = Rime, trad. Adelin Charles Fiorato, Paris, Les Belles Lettres, coll. «Bibliothèque italienne », 2004, p. 93.

13. Cette distinction est déjà présente chez Pline l'Ancien, chez Alberti et dans le principal traité de sculpture de la Renaissance avant Cellini, celui de Pomponio Gaurico, cf. De Statua, in P. Barocchi, Scritti d'arte del Cinquecento, Milan, R. Ricciardi, 1971, vol. 2, p. 1162-1664.

14. Rudolf Wittkower, Qu'est-ce que la sculpture?: principes et procédures, trad. Béatrice Bonne, Paris, Macula, coll. « Histoire de l'art », 1995, p. 124. Nous suivons ici la description très précise qu'il a donnée du travail de Michel-Ange.

15. Giorgio Vasari, Les Vies des meilleurs peintres, sculpteurs et architectes, trad. André Chastel, Paris, Berger-Levrault, 1981, vol.1, p. 124.

16. Id. Pour l'italien, nous utilisons l'édition numérique en ligne.

17. Rudolf Wittkower, Qu'est-ce que la sculpture?, op. cit., p. 136.

18. Rappelons la définition que donne Vasari du disegno : « [il] est donc l'expression sensible, la formulation explicite d'une notion intérieure à l'esprit ou mentalement imaginée par d'autres et élaborée en idée ", Giorgio Vasari, Les Vies, op. cit., vol.1, p.149. À la différence de Vasari qui 
considère que le disegno est composé à partir de différentes formes observées, Michel-Ange forme le disegno en gardant à l'esprit les caractéristiques du bloc de marbre.

19. Ibid., p. 150.

20. Leonard Robert Rogers, Comprendre la sculpture, trad. Arnaud Guilloux et Gérard Le Don, Rennes, Presses Universitaires de Rennes, 2015, p. 178.

21. Michel-Ange, Carteggio = Correspondance, op. cit., vol. 1, p. 166.

22. Léonard de Vinci, Traité de la peinture, trad. André Chastel, Paris, Calmann-Lévy, 2003, p. 102.

23. Pour ce détail, voir : Benvenuto Cellini, Traités de l'orfèvrerie et de la sculpture, op. cit., p. 168.

24. Rudolf Wittkower, Qu'est-ce que la sculpture ?, op. cit., p. 123.

25. Ascanio Condivi, Vie de Michelangelo Buonarroti, op. cit., p. 94.

26. Leonard Robert Rogers, Comprendre la sculpture, op.cit., p.189. L'auteur précise: «Les sculptures taillées semblent souvent contenues dans les limites d'un espace qui leur est propre, une capsule d'espace correspondant en gros à la forme du matériau dans lequel elles ont été taillées, alors que les sculptures modelées établissent des relations beaucoup plus libres avec l'espace environnant [...].» Cette impression vient des connexions qui existent entre les différents points de saillies qui se trouvent sur le même plan.

27. Erwin Panofsky, «Le mouvement néoplatonicien et Michel-Ange» in Essais d'iconologie: thèmes humanistes dans l'art de la Renaissance, trad.Claude Herbette, Paris, Gallimard, coll. «Bibliothèque des sciences humaines", 1967, p. 260. Panofsky y participe à une discussion décisive concernant la sculpture chez les historiens allemands de l'art du début du $\mathrm{xx}^{\mathrm{e}}$ siècle : une sculpture en ronde-bosse, indépendante, vue de face, traitée sur le mode du relief, est-elle identique à une image bidimensionnelle? Le débat avait été lancé par Hildebrand, qui soutenait qu'une telle sculpture avait les caractéristiques d'une «image plate» (Adolf Hildebrand, Le Problème de la forme dans les arts plastiques, trad. Éliane Beaufils, Paris, L'Harmattan, coll. «Esthétiques», 2002, p. 75), position que Panofsky nuance au regard de la sculpture de Michel-Ange.

28. Poésies $=$ Rime, op. cit., p. 93. Nous traduisons.

29. Le commentaire de Varchi, Lezzione sopra un sonetto di Michelangelo, a été reproduit dans $P$. ${ }^{\circ}$ Barocchi, Scritti d'arte, op. cit., vol. 2, p. 1322-1341. Pour une analyse de ce texte, notamment en lien avec le paragone, se rapporter à : Luciano Migliaccio, «Poemas de mármore: Michelangelo escultor e poeta nas Lezioni de Benedetto Varchi », Revista Brasileira de História, 1998, vol. 18, n 35 , p. 207-216.

30. Errico Vitale, "Sul concetto di materia nella Theologia Platonica di Marsilio Ficino", Rinascimento, 1999, vol. 39. Nous ne prétendons pas dans cet article reconstituer l'ensemble de la pensée de Ficin consacrée à la matière. On pourra pour cela se rapporter également à l'article de Tristan Dagron, "Ficin et la conception néoplatonicienne de la matière " in Marsile Ficin ou Les mystères platoniciens: actes du $42^{e}$ colloque international d'études humanistes [Centre d'études supérieures de la Renaissance], Tours, 7 au 10 juillet 1999, Stéphane Toussaint (dir.), Paris, Les Belles Lettres, 2002, p. 163-177. Ou encore à : James G. Snyder, « The Theory of materia prima in Marsilio Ficino's Platonic Theology ", Vivarium, 2008, vol. 46, n² 2, p. 192-221.

31. Marsile Ficin, Théologie platonicienne de l'immortalité des âmes, trad. Raymond Marcel, Paris, Les Belles Lettres, 1964, vol.3, livre XVII, chapitre 2, p. 149. Nous modifions l'ensemble des traductions de Ficin.

32. Ibid., livre XI, chapitre 3, p. 108.

33. On trouvera aisément des formules semblables chez Pic de la Mirandole, comme dans son Commento sopra una canzone d'amore di Girolamo Benivieni (Commento, trad. Stéphane Toussaint, Lausanne, L’Âge d'homme, coll. «Contemplation », 1989, livre II, chapitre XIV, p. 121.) : « [...] chaos ne signifie rien d'autre que la matière pleine de toutes les formes, mais confuses et imparfaites ». Nous préférons nous attarder ici sur Ficin, d'abord par un souci de concision, mais également parce que chez lui le dynamisme de la matière et la coloration vitaliste de sa cosmologie nous 
semblent être plus proches de la manière dont Michel-Ange conçoit la matière. Pic de la Mirandole, au chapitre vi du De ente et uno, a cependant discuté la théorie ficinienne selon laquelle la matière, étant informe, est une et extérieure à l'être : «Et en effet Platon ne veut pas que la matière soit tout à fait rien, sans quoi comment sera-t-elle le réceptacle des formes, comment sera-t-elle la nourrice, dotée d'une nature et d'autres attributs, ainsi que Platon le confirme dans le Timée? ", « [la matière] n'est donc pas rien, elle n'est pas tout à fait en dehors de l'étant." (De ente et uno, chapitre VI, in Jean Pic de la Mirandole, Euvres philosophiques, trad. Olivier Boulnois et Giuseppe Tognon, Paris, Puf, coll. «Épiméthée», 2004, p. 114.) Le néoplatonisme florentin cherche à concilier trois exigences : celle, logique, selon laquelle une matière tout à fait informe ne saurait recevoir de forme ; celle, cosmologique, selon laquelle la matière demeure le dernier degré d'une hiérarchie de réalités ordonnées selon la présence plus ou moins actualisée de la forme ; et celle, théologique, selon laquelle l'ensemble des réalités, y compris la matière, doit manifester, ne serait-ce qu'à un degré infime, l'éclat de la bonté divine.

34. Marsile Ficin, Théologie platonicienne, op. cit., vol. 1, livre III, chapitre 4, p. 176-177.

35. Ibid., livre I, chapitre 6, p. 71.

36. Marsile Ficin, Commentaire sur le Banquet de Platon, trad. Raymond Marcel, Paris, Les Belles lettres, coll. « Les Classiques de l'humanisme », 1956, troisième discours, § 6, p. 264. Dans ce texte, Ficin définit la beauté comme vivacitas.

37. Giorgio Vasari, "Vie de Michel-Ange», Vies des artistes: vies des plus excellents peintres, sculpteurs et architectes, trad. Léopold Leclanché et Charles Weiss, Paris, B. Grasset, coll. «Les Cahiers rouges ", 2007, p. 424.

38. Rudolf Wittkower, Qu'est-ce que la sculpture ?, op. cit., p. 121.

39. Erwin Panofsky, Die Gestaltungsprincipien Michelangelos, besonders in ihrem Verhältnis zu denen Raffaels, Berlin, De Gruyter, 2014, p. 144-145. L'idée d'une poussée inhibée, décrite par Panofsky, est retrouvée d'une autre manière à propos des tensions inhérentes au Moïse par Daniel Arasse dans « L'index de Michel-Ange ", Communications, 1981, vol. 34, n 1, p. 6-24.

40. Michel-Ange, Poésies = Rime, op. cit., p. 124 .

41. Léonard de Vinci, Traité de la peinture, op. cit., p. 93.

42. Gaston Bachelard, La Terre et les rêveries de la volonté, Paris, José Corti, 1947, p. 17.

43. "l'inculta sua cruda e dura scorza ", Michel-Ange, Poésies = Rime, op. cit., p. 93.

44. "Argomentano ancora dalla lunghezza del tempo, dicendo che la scultura è quasi perpetua", Benedetto Varchi, Lezione, nella quale si disputa della maggioranza delle arti e qual sia più nobile, la scultura o la pittura, in Paola Barocchi, Trattati d'arte del Cinquecento fra Manierismo e Controriforma,

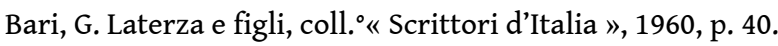

45. Marsile Ficin, Théologie platonicienne, op.cit., vol.1, livre XV, chapitre 8, p.180. Nous soulignons.

46. «Les œuvres de l'art demeurent sans corruption dans la mesure où elles sont conservées par la force de la nature comme une statue dure longtemps par la solidité naturelle de la pierre ou du bronze. De même les objets naturels demeurent dans la mesure où ils sont conservés par l'influx de Dieu », Ibid., livre II, chapitre 7, p. 94.

47. Baldassare Castiglione, Il Corteggiano, livre I, chapitre xxvI, in Baldassare Castiglione, Giovanni Della Casa et Benvenuto Cellini, Opere, Milan, R. Ricciardi, 1960, p. 47-48.

48. Michel-Ange, Carteggio = Correspondance, op. cit., p. 2, p. 65-66.

49. «Vie de Michel-Ange », op. cit., p. 357. Nous soulignons.

50. Voir sur cette question : Silke Schauder, «Figures de l'inachèvement : Michel-Ange et Camille Claudel », Topique, 30 octobre 2008, n 104, p. 173-190. Il faudrait compléter cette réflexion par une histoire de cette expression du non-finito souvent accolée à la pierre : redécouvrant les sculptures antiques abîmées par le temps, la Renaissance conçoit un goût singulier pour l'inachevé, comme en témoigne un poème de Laurent de Médicis : "Les membres montrent seulement une figure/ Esquissée et inachevée dans une pierre dure (bozzata et non finita in pietra 
dura)» (pour cette citation et une étude de cette expression, voir : Paul Barolsky, « As in Ovid, So in Renaissance Art », Renaissance Quarterly, 1998, vol.51, n², p. 451- 474.) Par ailleurs, André Chastel a établi une distinction essentielle entre le fragmentaire, qui à l'image de la ruine est une partie qui fait signe vers une forme totale absente et défaite, et le non-finito, qui est une forme qui n'a jamais été parachevée (Fables, formes, figures, op. cit., vol. 2, p. 39). Chastel propose également une interprétation métaphysique du non-finito, utilisé par Michel-Ange en contraste avec des formes bien polies : cela permet à Michel-Ange de «définir des zones mortes ou indistinctes à côté d'autres plus actives", de faire "jouer des degrés de réalité différents"; le non-finito "participe à l'effet total» (Art et humanisme, op.cit., p. 331.) La matière qui figure une forme ébauchée et confuse vient rehausser la forme totalement explicitée, et ces différences de finition permettent d'évoquer un cosmos hiérarchisé selon des degrés continus, comme dans le néoplatonisme florentin. On pourrait ajouter que Michel-Ange figure en même temps différents moments du travail.

51. Nous reprenons le texte italien de 1568. Dans cet ajout de la seconde édition de ses Vite, Vasari évoque la différence entre une œuvre complètement achevée de Luca della Robbia et une œuvre semblable, mais inachevée, de Donatello, et tranche finalement en faveur de la seconde.

52. Anecdote citée par Wittkower dans Qu'est-ce que la sculpture?, op. cit., p. 164.

53. Marsile Ficin, Théologie platonicienne, op. cit., vol. 1, livre IV, chapitre I, p. 150.

54. Ibid., livre IV, chapitre 7, p. 243.

55. Yves Hersant, « Le marteau de Michel-Ange », Communications, 1997, vol. 64, n 1, p. 85.

56. Voir exemplairement le cas de Vincenzo Danti, élève de Michel-Ange, qui distingue l'imitare, imitation des choses telles qu'elles sont, du ritrare, correction des imperfections : «l'ordre peut être plus parfait dans l'art que dans la nature, puisque l'homme, qui connaît les imperfections des choses naturelles, peut grâce à l'art les éviter et y remédier ", Il primo libro del trattato delle perfette proprorzioni. Di tutte le cose che imitare e ritrarre si possano con l'arte del disegno., in Paola Barocchi, Trattati d'arte, op.cit., p. 220. Dans le livre XIII de la Théologie platonicienne, Ficin soutient également que le rôle de l'homme est d'embellir la nature.

57. Michel-Ange, Poésies = Rime, op. cit., p. 26. Traduction modifiée : nous préférons traduire nonfinito par inachevé plutôt que par «imparfait». La comparaison entre l'artiste et Dieu est très fréquente à la Renaissance. Ficin faisait de l'homme un émule de Dieu, dont il n'était distingué que par la force et la matière sur laquelle il travaille : « qui pourrait nier que l'homme soit, pour ainsi dire, presque doté du même génie que le divin auteur des cieux, et qu'il pourrait, d'une certaine manière, faire les cieux, s'il trouvait des instruments et une matière céleste, après qu'il les ait rendus, bien qu'à partir d'une autre matière, cependant tout à fait semblables à l'ordre? » (Marsile Ficin, Théologie platonicienne, op. cit., vol. 2, livre XII, chapitre 3, p. 226.) Sur la place de l'art et de la création chez Ficin, voir: Caroline Combronde, "Les platoniciens de l'art à la Renaissance ", Revue Philosophique de Louvain, 1999, vol. 97, n² 2, p. 268-288.

58. Marsile Ficin, Théologie platonicienne, op. cit., vol. 1, livre IV, chapitre 2, p. 169.

59. Ibid., vol. 3, livre XV, chapitre 11, p. 64. Nous soulignons, en français.

60. Yves Hersant, « Le marteau de Michel-Ange », op. cit., p. 84.

61. Erwin Panofsky, Idea : contribution à l'histoire du concept de l'ancienne théorie de l'art, trad. Henri Joly, Paris, Gallimard, coll. « Tel », 1989, p. 143. Nous soulignons.

62. Le modelage se déploie hors du cadre de référence qu'est le bloc de marbre. Comme l'écrit Rogers dans Comprendre la sculpture, op. cit., p. 184 : « La sculpture figurée obtenue par modelage peut donc faire preuve d'un degré de liberté dans l'espace impossible à obtenir dans la pierre, à moins d'efforts énormes pour surmonter les limitations structurelles inhérentes à celle-ci. »

63. Giorgio Vasari, «Vie de Michel-Ange », op. cit., p. 424.

64. Ibid., p. 428. 
65. Erwin Panofsky, Idea, op. cit., p. 146. Cet abîme ne doit cependant pas être compris comme l'absence totale d'idéalité dans le sensible, mais comme ce qu'il reste à combler une fois découvertes les semences de forme que la matière contient.

66. "Si come nella penna e nell'inchiostro/ è l'alto e 'l basso e 'l mediocre stile/ e ne' marmi l'immagin ricca e vile/ secondo che 'l sa trar l'ingegno nostro", Michel-Ange, Poésies = Rime, op. cit., p. 53. De même Léonard écrit: "Nous dirons donc que le défaut est dans l'ouvrier non dans son matériau ", Léonard de Vinci, Traité de la peinture, op. cit., p. 101. Vincenzo Danti affirme quant à lui que l'imperfection peut venir ou bien de la matière ou bien de l'artiste, et notamment de la façon dont il a conçu l'œuvre. Il semble que les artistes aient opté pour la deuxième solution, que Danti décrit en ces termes : « d'autres fois encore, la matière sera apte à recevoir la perfection du composé de la figure, mais l'artiste ne sera pas apte à composer à la perfection l'idée (idea) de la figure dans son intellect et à la réaliser ", Trattato delle perfette proprorzioni, in Trattati d'arte, op. cit. ,p. 262.

67. "The artist was finally alienated because his imagination was by definition outside the God-given order of the world and its exercise led inevitably to the sin of pride ", David Summers, Michelangelo and the language of art, Princeton, Princeton University Press, 1981, p. 458.

68. Giorgio Vasari, "Vie de Michel-Ange », op. cit., p. 425. Pour le lien entre difficoltà et terribiltà, voir : David Summers, Michelangelo, op. cit., p. 234.

69. Michel-Ange, Carteggio = Correspondance, op. cit., vol. 2, p. 12.

70. Plotin, Traités. 1-6, trad. Francesco Fronterotta, Jérôme Laurent, Laurent Lavaud et Alain Petit, Paris, Flammarion, 2002, p. 79.

71. Ange Politien, lettre à Paulo Cortesio in E. Garin, Prosatori latini del Quattrocento, Milan, R. Ricciardi, 1952, p. 902.

72. Marsile Ficin, Théologie platonicienne, op.cit., vol.2, livre X, chapitre IV, p.69. Le terme designatur annonce le disegno de Vasari. Voir sur ce point : Thierry Gontier, « Noétique et poièsis : L'idea dans la Theologia platonica de Marsile Ficin », Archives de Philosophie, 2004, n 1, p. 5-22. Ce texte doit également être comparé avec un extrait du De Amore, dans lequel Ficin écrit que, lorsque le sensible reçoit la beauté, « la forme de l'homme que l'âme possède apparaîtra dans une matière harmonieuse et obéissante de manière plus expressive (expressior)» (Marsile Ficin, Commentaire sur le banquet, op. cit., sixième discours, § 1, p. 200).

73. Jean Pic de la Mirandole, Euvres philosophiques, op. cit., p. 270.

74. Marsile Ficin, Théologie platonicienne, op. cit., vol. 3, livre XVI, chapitre 2, p. 118.

75. On trouvera une telle remise en cause chez l'anthropologue britannique Tim Ingold, qui s'est penché sur l'histoire de l'architecture pour penser le faire (making) artistique comme un "processus de croissance », qui constitue une intervention dans une matière déjà en processus de changement. Il écrit : "This is to place the maker from the outset as a participant in amongst a world of active materials. These materials are what he has to work with, and in the process of making he "joins forces" with them [...]. Far from standing aloof, imposing his designs on world that is ready and waiting to receive them, the most he can do is to intervene in worldly processes that are already going on " (Making: Anthropology, Archaeology, Art and Architecture, Londres, Routledge, 2013, p. 21.) La Renaissance, sans directement remettre en cause le modèle hylémorphique, avait pensé une matière plus « active »; et la sculpture de Michel-Ange rapproche le faire d'un tel processus de croissance. 


\section{RÉSUMÉS}

Le présent article propose une interprétation philosophique d'un geste artistique, la scultura per forza di levare de Michel-Ange, en le replaçant dans son contexte philosophique et artistique. En procédant par soustraction et non par ajout, la sculpture de Michel-Ange congédie l'idée selon laquelle la création artistique serait nécessairement un travail de mise-en-forme d'une matière informe. Elle invite à penser une matière vivante, suggestive de formes et semble par là achever certaines intuitions du néoplatonisme florentin. Mais la sculpture de Michel-Ange conduit également à réfléchir sur la temporalité propre au travail du sculpteur, sur la résistance d'une matière dure à laquelle l'artiste doit se confronter.

INDEX

Mots-clés : art, métaphysique, matière, forme, sculpture, Ficin, pensée de la Renaissance

\section{AUTEUR}

\section{BAPTISTE TOCHON-DANGUY}

Philosophe agrégé, Élève de l'École Supérieure de Lyon, baptiste.tochon@ens-lyon.fr 